\title{
Routine multipurpose gas chromatographic assay for urinary corticosteroids
}

\author{
D. MURPHY AND H. F. WEST \\ From the Rheumatism Research Unit, Nether Edge Hospital, Sheffield
}

SYNOPSIS The use of a routine assay for urinary steroids is described that is thought to satisfy the following needs: the measurement of low levels of 17-hydroxycorticosteroid excretion; the assess $=\overline{\bar{g}} \mathrm{O}$ ment of adrenocortical activity during prednisolone therapy; the diagnosis of the adrenogenitaliv syndrome and the monitoring of its treatment using any available urine specimen; the very early $\vec{\omega}$ diagnosis of pregnancy using any available urine specimen. It may also prove of value in the assessment of progesterone secretion and metabolism during pregnancy.

Corticosteroid assays are used for the measurement of hypo- and hyperfunction of the adrenal cortex; the assessment of iatrogenic adrenal suppression; the measurement of the degree of adrenal stimulation induced with ACTH; the diagnosis of the adrenogenital syndrome and the assessment of its treatment. If a single quick routine assay could provide unequivocal information for each of these needs and could provide, in addition, an early dignosis of pregnancy and an assessment of the excretion of a number of pregnancy steroids it would be of considerable use. This paper describes the clinical use of an assay that appears to provide such information.

\section{METHODS}

Details of the routine assay procedure for urinary 17-hydroxycorticosteroids (17-OHCS) have been published (Murphy and West, 1966). In brief, $10 \mathrm{ml}$ of urine (or much less, in special circumstances, made up to $10 \mathrm{ml}$ with distilled water) is adjusted to $p \mathrm{H} 7$ and $1 \mathrm{ml}$ of potassium borohydride solution $(15 \%$ in $0.1 \mathrm{~N} \mathrm{NaOH})$ added. After heating at $60^{\circ} \mathrm{C}$ for $50 \mathrm{~min} 1 \mathrm{ml}$ of $25 \%$ acetic acid is added followed, in $2 \mathrm{~min}$, by $4 \mathrm{ml}$ of sodium metaperiodate solution (10\% aqueous) and the heating continued for a further 10 minutes. One $\mathrm{ml}$ of $5 \mathrm{~N} \mathrm{NaOH}$ is then added and the products extracted with $20 \mathrm{ml}$ of ethylene dichloride. The extract is filtered through sodium sulphate and evaporated to dryness before acetylation at $60^{\circ} \mathrm{C}$ for 30 min with $0.2 \mathrm{ml}$ of pyridine and $0.1 \mathrm{ml}$ of acetic anhydride. The reagents are then evaporated under nitrogen and the residue dissolved in $0.4 \mathrm{ml}$ ethanol for gas chromatography. When it has been necessary to obtain more information by forming trimethylsilyl ether (TMSi) derivatives, of the C,3 and C,17 hydroxyl functions, this was effected as follows. The dry unacetylated

Received for publication 25 October 1967. extracts were reacted with $0.2 \mathrm{ml}$ of purified pyridine and $0.1 \mathrm{ml}$ of hexamethyldisilazane overnight at room ${ }^{*}$ temperature. The reagents were then evaporated under $\overrightarrow{0}$ nitrogen and the residue was dissolved in $0.4 \mathrm{ml}$ of ethylene dichloride ready for gas chromatography. The acetates were gas chromatographed at $235^{\circ} \mathrm{C}$ and theo TSMi ether derivatives at $210^{\circ} \mathrm{C}$. In the routine $17-\mathrm{OHCS}$ assay the major $\mathrm{C}, 21$ metabolites of cortisol, ie, the glucosiduronates of tetrahydrocortisol, tetrahydrocorti-ڤ sone, the cortols, the cortolones and their $5 a$ isomers ${ }_{Q}$ are converted to $11 \beta$-hydroxyactiocholanolone and $11 \beta-\vec{z}$ hydroxyandrosterone and these are measured together윽 in a single gas chromatograph peak. Pregnanetriol is converted to aetiocholanolone acetate and pregnanediol to its diacetate. In the assay the retention times are approximately $12 \mathrm{~min}$, four $\mathrm{min}$, and six $\min$ respec- $\overline{0}$ tively.

\section{RESULTS}

The first 'normal' gas chromatogram of Fig. 10 shows the usual appearance of the peak derived from urinary 17-hydroxycorticosteroids (17-OHCS)o in 1/8000th of a $24-\mathrm{hr}$ specimen. The second 'normal' gas chromatogram shows a subsidiary peak on the right hand side of the 17-OHCS peak. This peak has N been found to be derived from, and to give a measure of, the 11-oxygenated 17-ketosteroids derived from cortisol (unpublished). The third gas chromatogram labelled PP $5 \mathrm{mg}$, is from 1/8000th of a 24-hr: specimen of a patient receiving $5 \mathrm{mg}$ of prednisolone phosphate daily. The peak represents an excretion of $4.3 \mathrm{mg}$ of 17 -hydroxycorticosteroids. (The normalō is $10 \mathrm{mg} \pm 3$ and varies with the muscle mass of the $\vec{\otimes}$ subject.) The degree of adrenal suppression caused $\frac{\text { के }}{0}$ by $5 \mathrm{mg}$ of prednisolone phosphate was found to vary from patient to patient. In an average-sized 


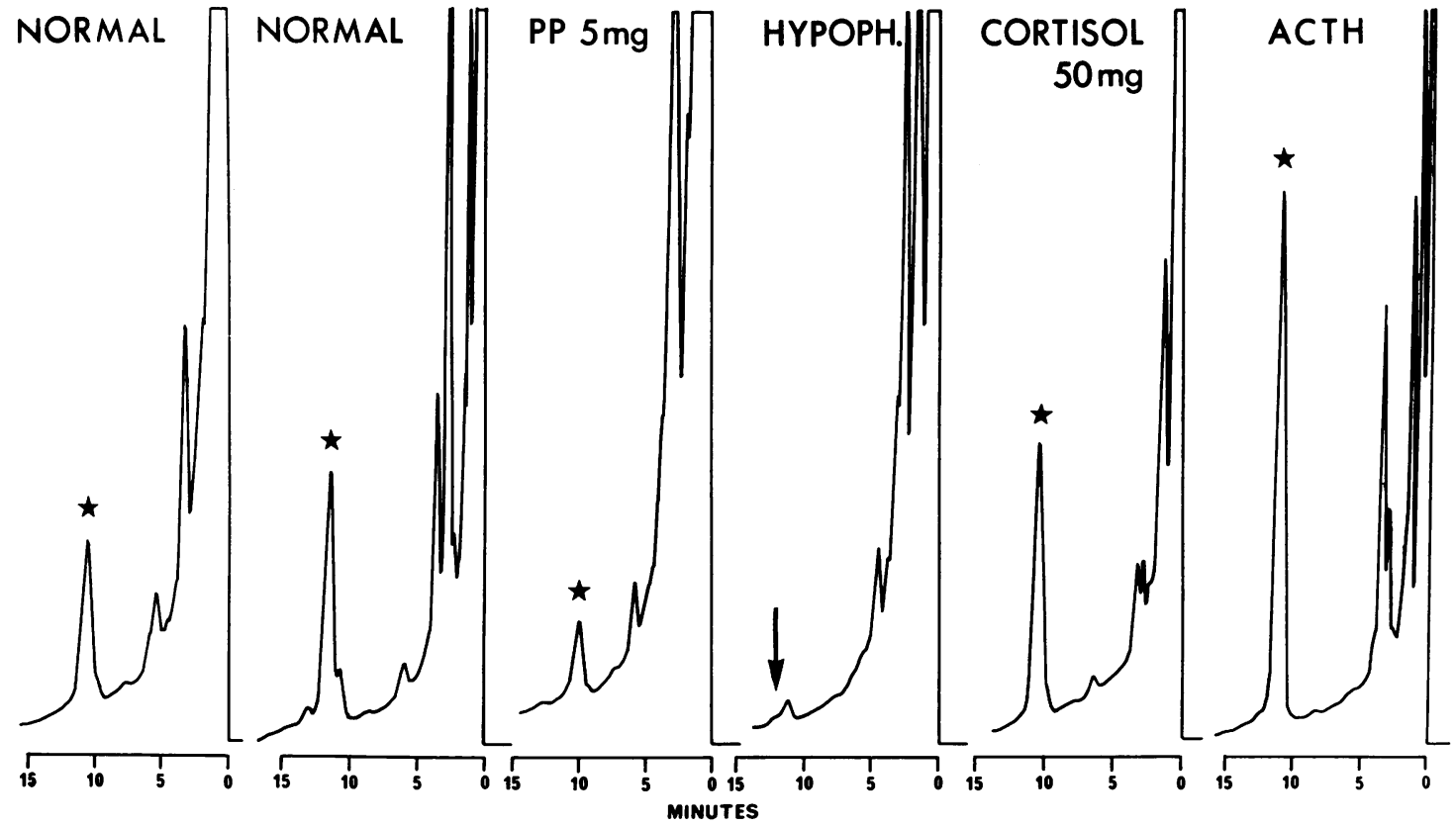

FIG. 1. Gas chromatograms from extracts of 24-hr urine specimens. The star marks the peak derived from 17hydroxycorticosteroids and the arrow points to the position at which the 17-OHCS peak should appear. Each chromatogram was run for at least 20 min and no other peaks appeared.

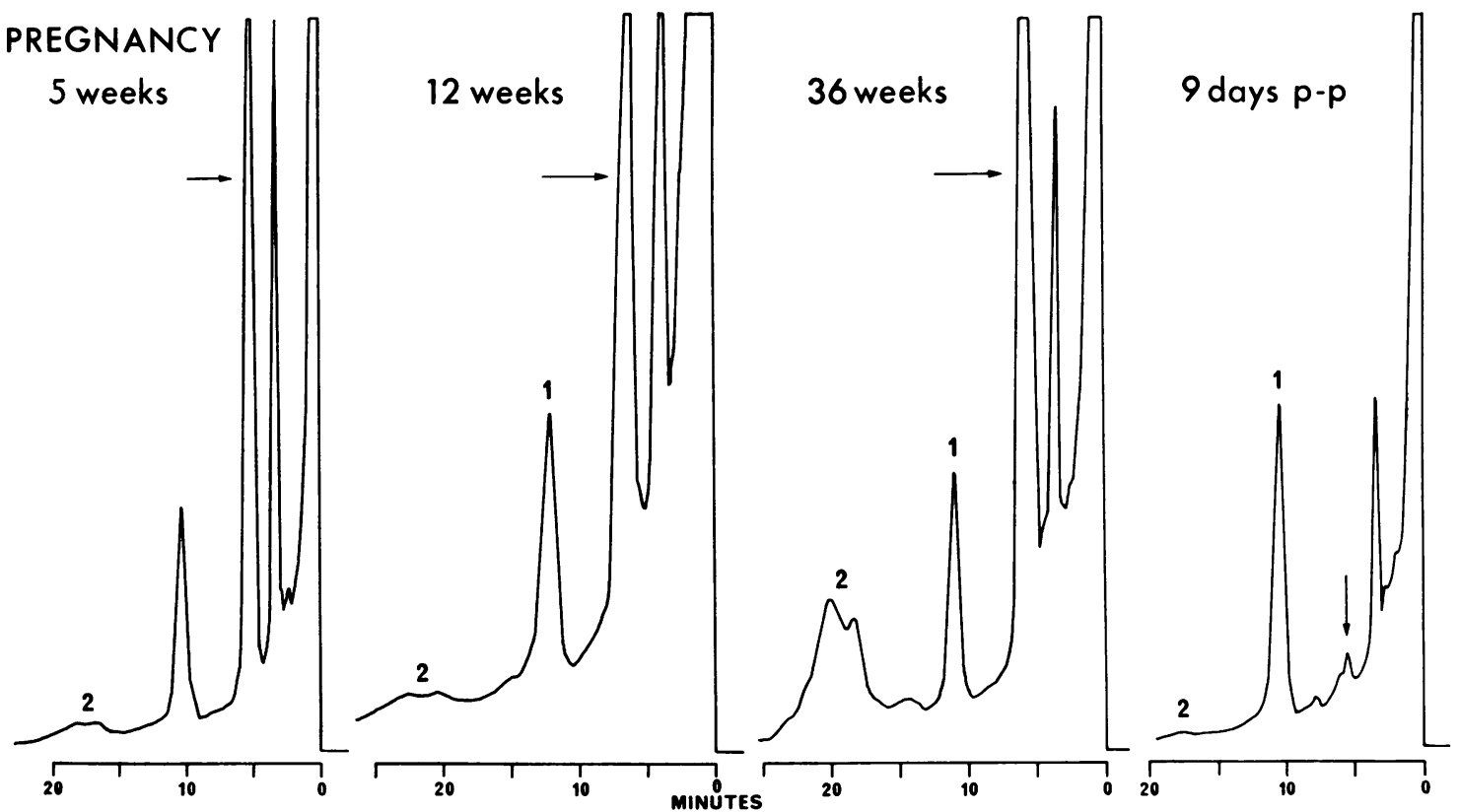

FIG. 2. Gas chromatograms of extracts of 24-hr urine specimens. The weeks are counted from the last period. One 8000th of each specimen was chromatographed. 


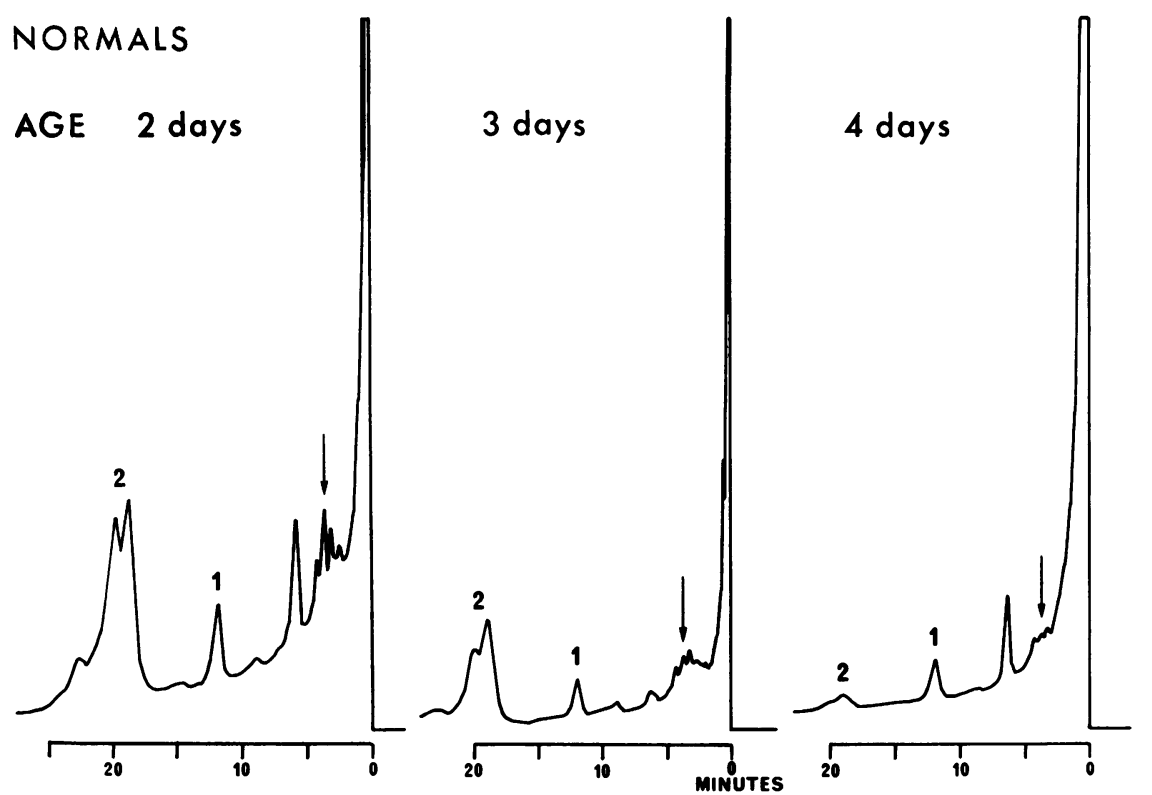

14 days

FIG. 3. Gas chromatograms of extracts of urine specimens obtained from babies. The quantities of urine chromatographed were, from left to right $0.03,0 \cdot 156,0.25$, and $0.25 \mathrm{ml}$ respectively.
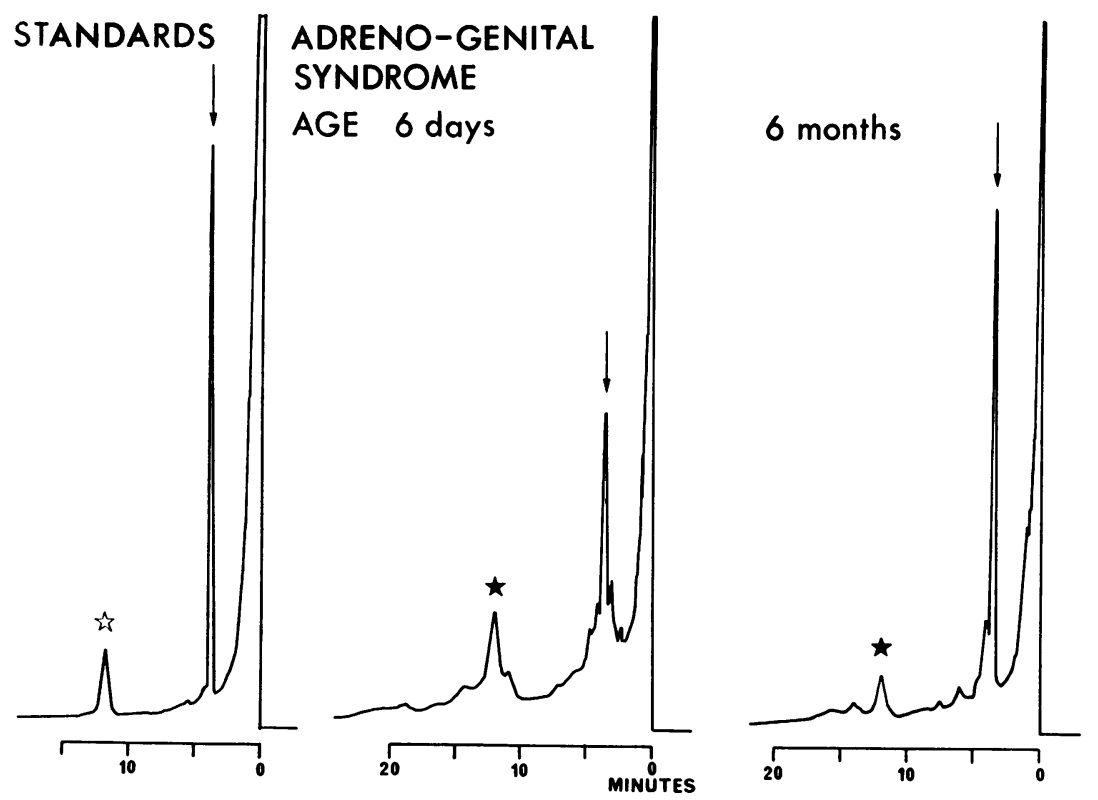

14 years

FIG. 4. Gas chromatograms of extracts of standards and of urine from female children with abnormal external $\stackrel{\mathbb{D}}{\Omega}$ genitalia. The arrows of the children's chromatograms mark peaks with the retention time of pregnanetriol. The 0 quantities of urine chromatographed were, from left to right, $0.06 \mathrm{ml}, 0.06 \mathrm{ml}$, and 1/7680 0 of a 24-hr specimen. 
active patient there was hardly any suppression. The fourth gas chromatogram of Fig. 1, labelled HYPOPH, is from a hypophysectomized patient receiving prednisolone. The arrow points to where the 17-OHCS peak would be. The fifth gas chromatogram, labelled CORTISOL, is of $1 / 16000$ th of a 24-hr specimen from a patient receiving $50 \mathrm{mg}$ of cortisol daily. The $17-\mathrm{OHCS}$ excretion was $21 \cdot 3 \mathrm{mg}$. The sixth gas chromatogram is $1 / 16000$ th of a $24-\mathrm{hr}$ specimen from a patient receiving ACTH. The 17-OHCS excretion was $42.9 \mathrm{mg}$.

Figure 2 shows gas chromatograms from 24-hr urine specimens collected during pregnancy. The fifth week specimen was from a subject with a regular 28-day cycle who was delivered 36 weeks after the specimen was taken. It will be seen that a complex of peaks labelled 2 has appeared and a peak designated by an arrow, that is, given by pregnanediol, is already high. Peak 1 is the 17-OHCS peak. In the non-pregnant woman we have not found the pregnanediol peak to exceed half the area of the 17-OHCS peak. The gas chromatogram labelled 12 weeks, 36 weeks, and nine days PP (post partum) were from a second subject. It will be seen that the complex peak and the 'pregnanediol' peak have virtually disappeared nine days after delivery. The large complex peak, which is peculiar to pregnancy, is thought to be derived from polar progesterone metabolites.

Figure 3 shows the assay procedure applied to babies' urine. The four gas chromatograms were selected from those of 30 babies to be representative of the change seen in the first week of life. The arrows mark the position at which a peak appears for pregnanetriol. Peak 1 is the 17-OHCS peak and peak 2 is similar to that seen in the mother's urine during pregnancy. This latter peak was still visible at 7 days in some cases.

Figure 4 shows on the left a gas chromatogram from standard pregnanetriol (arrow) and standard tetrahydrocortisone (star) put through the assay procedure. The other three gas chromatograms are from the only three cases of the adrenogenital syndrome that we have encountered. It will be seen that the pregnanetriol peak is greatly raised relative to the 17-OHCS peak. This latter peak is marked with a closed star as it does not consist solely of 17-OHCS in the adrenogenital syndrome. Fehér and Koref (1967) found the quantity of 11-oxygenated pregnanetriol metabolites, which will appear in the 17-OHCS peak, to be approximately one fourth of that of the pregnanetriol excreted.

\section{DISCUSSION}

The procedure has been in regular use here for 18 months and approximately 1,000 specimens have been assayed. A batch of specimens can be prepared for gas chromatography in two-and-a-half hours. A particular advantage of gas chromatographic assays is that they provide, in permanent form, the general pattern of steroid excretion as well as peaks for specific steroids. This pattern often provides confirmatory evidence of the validity of the measurement and sometimes unexpectedly reveals new peaks which the technique of gas chromatography helps one to track down and identify. The context of a peak in question may allow a condition to be diagnosed without the need for absolute measurement. As has been mentioned above, and as is evident from Figs. 2, 3, and 4, both the pregnancy gas chromatographic pattern and the adrenogenital syndrome gas chromatographic pattern can be obtained from a few millilitres of any unmeasured specimen that happens to be available.

A disadvantage of gas chromatography for quantitative assays is that unless the approximate quantity of the steroid to be measured is known it may be necessary to run a second chromatogram. Needless to say two or three chromatograms may be necessary to measure peaks that differ greatly in size, eg, the 17-OHCS peak and the pregnanediol peak in pregnancy.

One of the most useful derivatives for gas chromatography are the trimethylsilyl ethers. When these derivatives are substituted for the acetate derivatives the 17-OHCS peak is seen to be divided quantitatively into $5 \alpha$ and $5 \beta 17-\mathrm{OHCS}$ and it is possible to confirm that the peak measured as pregnanetriol is in fact pregnanetriol. It may be noted that on theoretical grounds all the more rare forms of the adrenogenital syndrome should give grossly abnormal gas chromatographic patterns. With regard to the specificity of the assays, it is possible that drugs may interfere. So far, with the exception of metapyrone, no such interference has been noted though additional peaks, not near the 17-OHCS peak, have been observed following the administration of some analgesics (not aspirin) and anabolic hormones. With the continuous advent of new drugs it is unwise to state that any assay for a given steroid is specific. It is our impression that when interference occurs it is more likely to be spotted in a gas chromatographic assay than in assays based on the development of colour or fluorescence.

Thanks are due to the Medical Research Council for financial support.

\section{REFERENCES}

Fehér, T., and Koref, O. (1967). Europ. J. Steroids, 2, 3. Murphy, D., and West, H. F. (1966). J. Endocr., 36, 331. 